\section{PTH-033 PATIENTS WITH CROHN'S DISEASE REPORT HIGH LEVELS OF FATIGUE DURING A SYMPTOMATIC DISEASE FLARE}

doi:10.1136/gut.2011.239301.434

C A Lamb, ${ }^{1, *}$ M Price, ${ }^{2}$ D E Jones, ${ }^{1}$ J C Mansfield ${ }^{3}$ IInstitute of Cellular Medicine, Newcastle University, Newcastle upon Tyne, UK; ${ }^{2}$ Gastroenterology, Newcastle upon Tyne Hospitals NHS Foundation Trust, Newcastle upon Tyne, UK; ${ }^{3}$ Gastroenterology, Newcastle University, Newcastle upon Tyne, UK

Introduction The symptom of fatigue can have a major impact on quality of life and is often overlooked by physicians. Previous research has indicated a significant link between fatigue and a number of chronic inflammatory conditions including primary biliary cirrhosis and multiple sclerosis. Patients with fatigue often experience sleep disturbance and autonomic nervous system dysregulation. This patient survey aimed to investigate the perceived severity of fatigue and other non-gastrointestinal systemic symptoms during a flare of Crohn's disease.

Methods Patients with Crohn's disease attending the Inflammatory Bowel Disease clinic were asked to complete a questionnaire scoring the severity of gastrointestinal, fatiguerelated and autonomic symptoms during a flare of Crohn's disease. As a hypothesised negative comparator they were also asked to score on urinary incontinence, not typically associated with Crohn's disease. Score 1 = not affected by Crohn's disease flare, Score 10 = severely affected by Crohn's disease flare. The results are summarised in table 1.

Results 17 patients (7 male and 10 female) completed the survey. The mean patient age was 32.4 years (range 19-53 years), with a mean duration of disease of 9.5 years (range 1-32 years) and a mean of 1.3 prior operative interventions (range $0-5$ ). Seven patients $(41.2 \%)$ had either a current or previous history of anti-TNF antibody use.

Table 1 PTH-033 Perceived symptom severity during a flare of Crohn's disease (mean symptom score $(95 \% \mathrm{CI})$ )

\begin{tabular}{lr}
\hline Abdominal pain & 8.4 (7.1 to 9.6$)$ \\
Diarrhoea & 8.2 (7.1 to 9.4$)$ \\
Fatigue & 6.8 (5.7 to 8.0) \\
Sleep disturbance & 6 (4.4 to 7.6$)$ \\
Concentration & $4.2(2.6$ to 5.8$)$ \\
Dizziness & $4(2.4$ to 5.6$)$ \\
Memory & $3.3(1.8$ to 4.8$)$ \\
Muscle pain & $3.6(1.9$ to 5.4$)$ \\
Bone pain score & $3.7(1.9$ to 5.4$)$ \\
Urinary incontinence & $1.3(0.3$ to 2.2$)$ \\
\hline
\end{tabular}

Conclusion Predictably, patients score abdominal pain and diarrhoea as severely affected by a flare of Crohn's disease. Interestingly fatigue and sleep disturbance were also perceived by patients to be highly affected by Crohn's disease activity. Interventions in Crohn's disease are often designed to impact on symptoms of abdominal pain and diarrhoea. Development of strategies to improve non-gastrointestinal symptoms including fatigue and sleep disturbance in active Crohn's disease may lead to a positive effect on quality of life. This data supports further investigation into the prevalence, biological causes and consequences of fatigue in Crohn's disease.

Competing interests None.

Keywords Crohn's disease, fatigue. 